J. Edu. \& Sci., Vol. 28, No. 2 , 2019 : 282-288

i- الدول النوال المستمرة من النوع

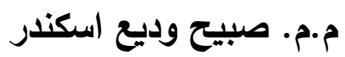 \\ قسم الرياضيات/كلية التربية للعلوم الصرفة/جامعة الموصل \\ الموصل/العراق \\ sabeehqaqus@yahoo.com \\ تاريخ الاستلام ت اريخ القبول \\ 2018/06/07 2018/02/25
}

الخلاصة

في هذا البحث نبرهن بان الدالة

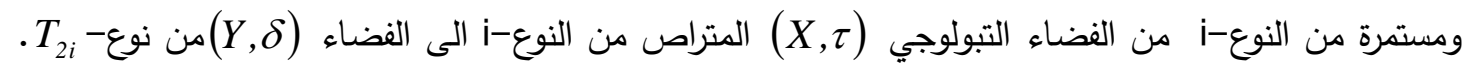

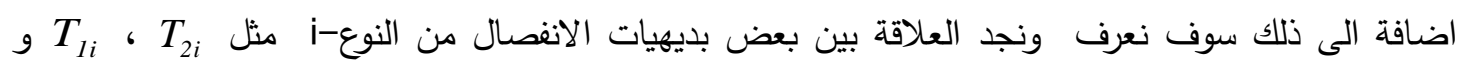
.$T_{\text {oi }}$

الكلمات المفتاحية: الدالة المستمرة من النوع-i ، التراص من النوع-i ، الدالة المفتوحة من النوع-i. 


\title{
On i-Continuous Functions
}

\author{
Sabih W. Askandar \\ Department of Mathematics \College of Education For Pure Science \\ University of Mosul \\ Mosul-Iraq \\ sabeehqaqus@yahoo.com
}

\section{Received Accepted \\ 25/02/2018 07/06/2018}

\begin{abstract}
In this paper we prove that the function $f:(X, \tau) \rightarrow(Y, \delta)$ is $\mathrm{i}$-open if it is injective, surjective and $\mathrm{i}$-continuous from $\mathrm{i}$-compact topological space $(X, \tau)$ into $T_{2 i}$-space $(Y, \delta)$. Further, we define and find the relationship among some i-separation axioms such as $T_{2 i}, T_{1 i}$ and $T_{\circ i}$.
\end{abstract}

Keywords: i-continuous function, i-compactness, i-open function.

\section{INTRODUCTION:}

It is well known that a continuous function on topological spaces needs not to be open. In [7] was proved that a continuous function which is injective and surjective from compact topological space into $T_{2}$-space is open. In 1987[4], Gangster, M. has defined strongly compact spaces and semi-compact spaces by using the concept of semi-open sets [5]. In 2011[2], Benchalli, S.S. and Neeli, U.I., are presented semitotal continuity. In 2012 [1], Askandar S.W., introduced the concept of i-open sets which he could to entire them together with many other concepts of Generalized open sets. In 2013[6], Mohammed, A.A and Askandar, S.W., introduced the relations between i-Separation Axiom, Semi-Separation Axioms and $\alpha$-Separation Axioms. In 2015[8],Tawfik, D.A. introduced g*i-closed sets in topological spaces. In 2018[3], Dembre, V., introduced new axioms in topological spaces called w- $T_{\mathrm{o}}, \mathrm{w}-T_{1}$ and w$T_{2}$-spaces. In this work, we generalize the result mentioned above by using the concept of i-open sets. The method used in the proving our results depend on the Pervin proof with some different important line, of this proof. Also we will find the relationship among Separation Axioms depend on i-open sets which we call iseparation axioms such as $T_{\circ i}, T_{1 i}$ and $T_{2 i}$. Throughout this work $(X, \tau),(Y, \delta)$ and $\left(X, \tau^{i}\right)$ always are topological spaces (where $\tau^{i}$ is a family of all i-open sets [1] of $\mathrm{X})$. This work consists of two sections. In the first one we begin by many useful concepts. In the second section we introduce the concept of i-compactness and use it to prove the main result and we have proved some important theorems to discuss the property of i-continuous functions(see Theorem 2.2, Theorem2.3, Theorem 2.4, Theorem 2.5, Theorem2.9, Theorem2.10 and Theorem2.11) and we find the relationship among some i-separation axioms(see Theorem 2.7). 


\section{Definitions and Examples.}

We begin in this section by the following useful concepts.

Definition1.1. A subset $A$ of a topological space $(X, \tau)$ is said to be i-open set[1] if there exists an open set $\mathrm{G} \neq \phi, \mathrm{X}$ such that $A \subseteq C l(A \cap G)$. The complement of an iopen set is called i-closed set. (Where $C l(A \cap G)$ denotes the closure of $(A \cap G)$ ).

Example1.2. Let $X=\{a, b, c, d\}, \tau=\{\phi,\{a\},\{c, d\},\{a, c, d\}, X\}$.

Open sets are: $\phi,\{a\},\{c, d\},\{a, c, d\}, X$, closed sets are: $X,\{b, c, d\},\{a$, $b\},\{\{b\}, \phi$. Take $A=\{a, b\}, G=\{a\}, A \cap G=\{a\}, C l(A \cap G)=\{a, b\}$.

So $A \subseteq C l(A \cap G)$. Then $\mathrm{A}$ is $\mathrm{i}$-open set but it is not open.

$A^{C}=\{c, d\}$ is i-closed set.

Definition1.3. Let $\left(X, \tau^{i}\right)$ be a topological space and let $A$ be a subset of $\mathrm{X}$. the intersection of all $\mathrm{i}$-closed sets containing $A$ is called i-Closure of $A[1]$, denoted by $C l_{i}(A): C l_{i}(A)=\bigcap_{i \in A} F_{i} . A \subseteq F_{i} \quad \forall i$ Where $F_{i}$ is i-closed set $\forall i$ in a topological space $\left(X, \tau^{i}\right) . C l_{i}(A)$ is the smallest $\mathrm{i}$-closed set containing $A, A=C l_{i}(A)$ if and only if $A$ is i-closed set.

Definition1.4. A function $f:(X, \tau) \rightarrow(Y, \delta)$ is said to be i-continuous[1] at the point $x_{o} \in X$ if and only if for each i-open set $\mathrm{I}^{*} \in \delta$ containing $f\left(x_{o}\right)$ there exists an i-open set $\mathrm{I}$ in $(X, \tau)$ containing $x$ 。 such that $f(\mathrm{I}) \subseteq \mathrm{I}^{*} . f$ is i-continuous map if it is $\mathrm{i}$-continuous at all points of $\mathrm{X}$.

Lemma1.5. [1] Let $f:(X, \tau) \rightarrow(Y, \delta)$ then the following conditions are each equivalent to i-continuity of $f$ on $\mathrm{X}$ :

i) The inverse of every $\mathrm{i}$-open set in $\mathrm{Y}$ is $\mathrm{i}$-open in $\mathrm{X}$.

ii) The inverse of every $\mathrm{i}$-closed set in $\mathrm{Y}$ is $\mathrm{i}$-closed in $\mathrm{X}$.

Definition1.6. A function $f:(X, \tau) \rightarrow(Y, \delta)$ is called i-open [1] if the image $f(O)$ of each i-open set $O$ in $(X, \tau)$ is i-open set in $(Y, \delta)$.

Definition1.7. A topological space $(X, \tau)$ is said to be $T_{\circ}$ space, if given any pair of distinct points $\mathrm{x}$, $\mathrm{y}$ of $X$, there exists an i-open set $I$, containing one of them but not the other (i-Klomogorov axiom).

Example1.8. Let $X=\{a, b\}, \tau=\{\phi,\{a\}, X\}, \tau^{i}=\{\phi,\{a\}, X\}$, are $\left(X, \tau^{i}\right)$ and $(X, \tau)$ topological spaces. Therefore; $a \in\{a\}, b \notin\{a\}$. s.t. $a, b \in X(a \neq b) \quad \exists\{a\} \in \tau^{i}$ . $T_{\circ i}$ is $(X, \tau)$

Definition1.9. A topological space $(X, \tau)$ is said to be $T_{1 i}$ space if for any two distinct points $\mathrm{x}$, $\mathrm{y}$ of $X$, there are an $\mathrm{i}$-open set $\mathrm{U}$ containing $\mathrm{x}$ but not $\mathrm{y}$ and $\mathrm{i}$-open set $\mathrm{V}$ containing $\mathrm{y}$ but not $\mathrm{x}$ ( $\mathrm{i}$-Frechet axiom). 
Example1.10. Let $X=\{a, b, c\}, \tau=\{\phi,\{a\},\{b\},\{c\},\{a, b\},\{a, c\}$,

$\{b, c\}, X\}=\tau^{i},\left(X, \tau^{i}\right)$ is a topological space.

$a, b \in X(a \neq b) \exists\{a\},\{b\} \in \tau^{i}$

s.t. $a \in\{a\}, b \notin\{a\}, b \in\{b\}, a \notin\{b\}$

$a, c \in X(a \neq c) \exists\{a\},\{c\} \in \tau^{i}$

s.t. $a \in\{a\}, c \notin\{a\}, c \in\{c\}, a \notin\{c\}$

$b, c \in X(b \neq c) \exists\{b\},\{c\} \in \tau^{i}$

s.t. $b \in\{b\}, c \notin\{b\}, c \in\{c\}, b \notin\{c\}$

Therefore; $(X, \tau)$ is $T_{1 i}$ - space.

Definition1.11. A topological space $(X, \tau)$ is said to be $T_{2 i}$-space if for any two distinct points $\mathrm{x}$, y of $X$, there exists two separated i-open sets $I_{1}$ and $I_{2}$ such that $I_{1}$ containing $\mathrm{x}$ and $I_{2}$ containing y ( $\mathrm{i}$-Hausdorff axiom).

Example1.12. Let $X=\{a, b\}, \tau=\{\phi,\{a\},\{b\}, X\}, \tau^{i}=\tau$

$(X, \tau)$ andare topological spaces. $\left(X, \tau^{i}\right)$

$a, b \in X(a \neq b) \exists\{a\},\{b\} \in \tau^{i}$

s.t. $a \in\{a\}, b \in\{b\},\{a\} \cap\{b\}=\phi$

Therefore; $(X, \tau)$ is $T_{2 i}$.

\section{2. i-Compactness and the Main Result.}

In this section we introduce the concept of i-compactness and use it to prove the main result also we find the relationship among $T_{2 i}, T_{1 i}$ and $T_{\circ i}$-space.

Definition2.1. A subset $A$ of a topological space $(X, \tau)$ is said to be i-compact if every cover of A by i-open sets has a finite sub cover, we call the topological space $(X, \tau)$ i-compact provided the set $\mathrm{X}$ is $\mathrm{i}$-compact.

Theorem2.2. Every i-closed subset of i-compact space is i-compact.

Proof: Let $A$ be i-closed subset of i-compact space $(X, \tau)$ and let $\left\{G_{k}\right\}$ be a cover of $A$ by i-open sets.

Now $\left\{G_{k} \cup A^{C}\right\}$ is a cover of $X$ by i-open sets, hence there exists a finite sub cover of X. i.e. $X \subseteq\left\{\left(\cup_{k=1}^{n} G_{k}\right) \cup A^{C}\right\}$ implies to $A \subseteq\left(\cup_{k=1}^{n} G_{k}\right)$. Therefore, $A$ is i-compact.

Theorem2.3. If $f$ is i-continuous function of $(X, \tau)$ into $\left(X^{*}, \tau^{*}\right)$ then $f$ maps every icompact subset of $X$ onto an i-compact subset of $X^{*}$.

Proof: Let $A$ be i-compact subset of $X$ and let $\left\{G_{k}^{*}\right\}$ be a cover of $f(A)$ by i-open sets. Since $A \subseteq f^{-1}(f(A)) \subseteq f^{-1}\left(\cup_{k} G_{k}^{*}\right)=\cup_{k} f^{-1}\left(G_{k}^{*}\right)$, the family $f^{-1}\left(G_{k}^{*}\right)$ is a cover of $A$ by i-open sets by (theorem 1.5). Since $A$ is i-compact, there must be some finite sub cover of $A$, 
say $A \subseteq\left(\cup_{k=1}^{n} f^{-1}\left(G_{k}^{*}\right)\right)$.

Now, $f(A) \subseteq f\left(\cup_{k=1}^{n} f^{-1}\left(G_{k}^{*}\right)\right)=\cup_{k=1}^{n} f\left(f^{-1}\left(G_{k}^{*}\right)\right) \subseteq \cup_{k=1}^{n} G_{k}^{*}$. Therefore, $\quad$ A is icompact.

Theorem2.4. Every i-compact subset $A$ of $T_{2 i}$-space $X$ is i-closed.

Proof: Let $\mathrm{x}$ be a fixed point in $A^{C}$. Since the space $\mathrm{X}$ is $T_{2 i}$, therefore; for each point $y \in A$, there exist two disjoint i-open sets $G_{x}$ and $G_{y}$ such that $x \in G_{x}$ and $y \in G_{y}$. The family of sets $\left\{G_{y}: y \in A\right\}$ is a cover of $A$ by i-open sets. Since $A$ is an i-compact, there must be some finite sub covering $\left\{G_{y_{k}}\right\}_{k=1}^{n}$. Let $\left\{G_{x_{k}}\right\}_{k=1}^{n}$ be the corresponding i-open sets containing $\mathrm{x}$ and $\operatorname{let} G=\cap^{n}{ }_{k=1} G_{x_{k}}$. Then $G$ is i-open set containing $\mathrm{x}$, we see that $G=\cap_{k=1}^{n} G_{x_{k}} \subseteq \cap_{k=1}^{n} G_{y_{k}}=\left(\cup_{k=1}^{n} G_{y_{k}}\right)^{C} \subseteq A^{C}$. Hence each point in $A^{C}$ is contained in i-open set which is itself contained in $A^{C}$. Therefore; $A^{C}$ is i-open set, so $A$ must be i-closed set.

Theorem2.5. Let $f$ is injective, surjective and i-continuous function from i-compact topological space $(X, \tau)$ into $T_{2 i}$-space $\left(X^{*}, \tau^{*}\right)$ then $\mathrm{f}$ is i-open.

Proof: Let $A$ be i-open set in $X$, so that $A^{C}$ is i-closed. By (theorem 2.2), $A^{C}$ is icompact. By (theorem 2.3), $f\left(A^{C}\right)$ is i-compact. By (theorem 2.4), $f\left(A^{C}\right)$ is i-closed. Since $f$ is injective and surjective, $f\left(A^{C}\right)=(f(A))^{C}$. so, as a consequence $f(A)$ is i-open set. Hence $f$ is i-open.

Example2.6. Let $\mathrm{X}=\{1,2\}, \tau=\{\phi,\{1\},\{2\}, \mathrm{X}\}, \mathrm{Y}=\{1,2\}, \delta=\{\phi,\{1\},\{2\}, \mathrm{Y}\}$,

$f:(X, \tau) \rightarrow(Y, \delta), f(1)=1, f(2)=2 f(3)=3$, It is clear that $(X, \tau)$ is i-compact space because $1,2 \in X$ and

$1 \in\{1\}, 2 \in\{2\}$ i.e. $X \subseteq\{1\} \cup\{2\}$, where $\{1\}$ and $\{2\}$ are $i$-open sets. so $\{1\} \cup\{2\}$ is a finite $\mathrm{i}$-open cover of $X$.

Also $(Y, \delta)$ is $T_{2 i}$-space because $1,2 \in X, 1 \neq 2$ there exist two i-open sets $\{1\}$ and $\{2\},\{1\} \cap\{2\}=\phi$ such that $l \in\{1\}, 2 \in\{2\}$.

$f$ is i-continuous function (Theorem 3.3). $f$ is i-open mapping (Definition 3.1).

Theorem2.7. Every $T_{2 i}$-space is $T_{1 i}$ and also is $T_{\circ}$.

Proof: 1 . Let $(X, \tau)$ be a $T_{1 i}$-space. Let a and b be two distinct points of $(X, \tau)$. Since $(X, \tau)$ is $T_{1 i}$-space, there exist two i-open sets $\mathrm{G}$ and $\mathrm{H}$ such that $a \in G, b \notin G$ and $a \notin H, b \in H$. Hence we have $a \in G, b \notin G$. Therefore $(X, \tau)$ is $\boldsymbol{T}_{\circ i}$-space.

2. Let $(X, \tau)$ be a $T_{2 i}$-space. Let $\mathrm{x}$ and $\mathrm{y}$ be two distinct points in $X$. Since $(X, \tau)$ is $T_{2 i}$-space, there exist two disjoint i-open sets $\mathrm{U}$ and $\mathrm{V}$ such that $x \in U, y \in V$. This implies, $x \in U, y \notin U, x \notin V, y \in V$. Hence $(X, \tau)$ is $T_{1 i}$-space.

3. From 1 and 2 we have, every $T_{2 i}$-space is $T_{\circ i}$.
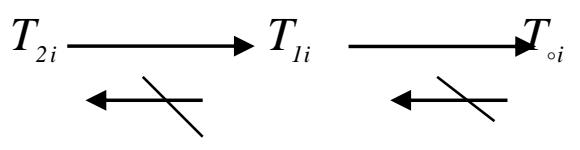
The converse of theorem $\mathbf{2 . 7}$ is not necessary to be true. Indeed,

Example2.8. Let $X=\{a, b, c\}, \tau=\{\phi,\{a\}, X\}, \tau^{i}=\{\phi,\{a\},\{a, b\},\{a, c\}, X\},\left(X, \tau^{i}\right)$ is a topological space.

$a, b \in X(a \neq b) \quad \exists\{a\} \in \tau^{i} \quad$ s.t. $a \in\{a\}, b \notin\{a\}$.

$a, c \in X(a \neq c) \quad \exists\{a\} \in \tau^{i}$ s.t. $a \in\{a\}, c \notin\{a\}$.

$b, c \in X(b \neq c) \quad \exists\{a, b\} \in \tau^{i}$ s.t. $\quad b \in\{a, b\}, c \notin\{a, b\}$. Therefore; $\quad(X, \tau) \quad$ is $T_{\circ i} .(X, \tau)$ is not $T_{1 i}$ - space, because,open -, there is no exist two i $a, b \in X(a \neq b)$ sets $\mathrm{G}$ and H s.t. $a \in G, b \in H$. Also, $(X, \tau)$ is not $T_{2 i}$ - space(definition 1.11).

Theorem2.9. Let $f:(X, \tau) \rightarrow(Y, \delta)$ be bijective i-open and i-continuous map from a $T_{\text {oi }}$-space $(X, \tau)$ onto a topological space $(Y, \delta)$. Then $(Y, \delta)$ is $T_{\circ}$-space.

Proof: Let a and $\mathrm{b}$ be two distinct points of $(Y, \delta)$. Since $f$ is bijective, there exist two distinct points $\mathrm{c}$ and $\mathrm{d}$ of $(X, \tau)$ such that $f(c)=a$ and $f(d)=b$. As $(X, \tau)$ is $T_{\text {o } i}$-space, there exists i-open set $\mathrm{G}$ such that $c \in G$ and $d \notin G$. Since $f$ is i-open map, then $f(G)$ is i-open in $(Y, \delta)$. As $f$ is i-continuous map we have $a \in f(G), \quad b \notin f(G)$. Hence $(Y, \delta)$ is $T_{\text {oi }}$-space.

Theorem2.10. If $f:(X, \tau) \rightarrow(Y, \delta)$ is a bijective i-open and i-continuous map from a $T_{1 i}$-space $(X, \tau)$ onto a topological space $(Y, \delta)$.Then $(Y, \delta)$ is $T_{1 i}$-space.

Proof: Let $(X, \tau)$ be a $T_{1 i}$-space. Let a and b be two distinct points of $(Y, \delta)$. Since $f$ is bijective, there exist two distinct points $\mathrm{c}$ and $\mathrm{d}$ of $(X, \tau)$ such that $f(c)=a$ and $f(d)=b$. Since $(X, \tau)$ is $T_{1 i}$-space, there exists i-open sets $\mathrm{G}$ and $\mathrm{H}$ such that $c \in G, d \notin G$ and $c \notin H, d \in H$. Since $f$ is i-open and i-continuous map, then $f(G)$ and $f(H)$ are i-open in $(Y, \delta)$ such that $a=f(c) \in f(G)$, $b=f(d) \notin f(G)$ and $a=f(c) \notin f(H), \quad b=f(d) \in f(H)$. Hence $(Y, \delta)$ is $T_{l i}$ space.

Theorem2.11. Let $f:(X, \tau) \rightarrow(Y, \delta)$ be a bijective i-open and i-continuous map. If $(X, \tau)$ is $T_{2 i}$-space then $(Y, \delta)$ is also $T_{2 i}$-space.

Proof: Let $(X, \tau)$ be $T_{2 i}$-space. Let $\mathrm{y}_{1}$ and $\mathrm{y}_{2}$ be two distinct points of $Y$. Since $f$ is bijective map, there exist two distinct points $\mathrm{x}_{1}$ and $\mathrm{x}_{2}$ of $X$ such that $f\left(x_{1}\right)=y_{1}$ and $f\left(x_{2}\right)=y_{2}$. Since $(X, \tau)$ is $T_{2 i}$-space, there exist i-open sets $\mathrm{G}$ and $\mathrm{H}$ such that $x_{1} \in G$, $x_{2} \in H$ and $G \cap H=\phi$. Since $f$ is i-open and i-continuous map, then $f(G)$ and $f(H)$ are i-open in $(Y, \delta)$ such that $y_{1}=f\left(x_{1}\right) \in f(G)$

$y_{2}=f\left(x_{2}\right) \in f(H)$ and $f(G) \cap f(H)=\phi$.

Therefore, $f(G) \cap f(H)=f(G \cap H)=\phi$. Hence $(Y, \delta)$ is $T_{2 i}$-space.

Acknowledgements: Mosul University. 


\section{References}

[1] Askandar, S.W., M.Sc. Thesis, College of Education, University of Mosul (2012).

(In Arabic)

[2] Benchalli, S.S. and Neeli, U.I., Continuous Functions in Topological Spaces, International Mathematical Forum, Vol.6, 2011, no.10, 479-492. [3]Dembre, V., New Axioms in Topological Spaces, International Journal of Computer Applications Technology and research, Volume7-Issue03, 109-113, 2018, ISSN: 2319-8656.

[4] Gangster, M., Some Remarks On Strongly Compact Spaces and Semi-Compact Spaces, Bull. Malaysian Math. Soc. (10)2(1987), 67-81.

[5] Levine, N., Semi-Open Sets and Semi-Continuity in Topological Space, Amer. Math. Monthly, 70(1963), 36-41.

[6]Mohammed, A.A. and Askandar, S.W., i-Open Sets and Separating Axioms Spaces , J. Education and Science Journal, College of Education for Pure Science, University of Mosul. (Accepted in march11, 2013)

[7] Pervin, W.J., Foundations of General Topology, Academic Press, London (1964).

[8] Tawfik,D.A., M.Sc., Thesis, College of Education, University of Mosul, (2015). (In Arabic) 